\title{
Interactive comment on "Brief Communication: \\ Conventional assumptions involving the speed of radar waves in snow introduce systematic underestimates to sea ice thickness and seasonal growth rate estimates" by Robbie D. C. Mallett et al.
}

\section{Ronald Kwok (Referee)}

ron.kwok@jpl.nasa.gov

Received and published: 4 October 2019

The comment was uploaded in the form of a supplement: https://www.the-cryosphere-discuss.net/tc-2019-198/tc-2019-198-RC1supplement.pdf 